DJS Vol. 42 (1) (2020) pp.108 - 124 ISSN: 1012-

\begin{tabular}{ccc}
\hline 1969 & Delta Journal of Science & Deta joumal of \\
\hline
\end{tabular}

Research Article

Geology

\title{
ASSESSMENT OF RAW MATERIALS FOR CEMENT INDUSTRY IN EL SUKHUNA-ZAAFRANA AREA, EASTERN DESERT, EGYPT
}

Hassan Z. Harraz; Mohamed M. Hamdy* , Abdel Salam M. Abu El-Ela, and Islam M. El-Hoseiny Geology Department, Faculty of Science, Tanta University, Tanta, Egypt

* Correspondence:

e-mail: mohamed.hamdy@science.tanta.edu.eg

\section{KEY WORDS}

Cement Industry; Raw

Materials; $\quad$ Red

Kaolinitic clay; Gebel El

Ramliya Limestone;

Mineral Resources,

Qiseib Formation, Abu

Darag Kaolinitic Clay,

Zaafrana Area, Eastern

Desert, Egypt

\section{ABSTRACT}

In the last few years, cement industry becomes a major player in materials recycling, although it is environmental polluted industry and a great consumer of limestone and clay. Cement manufacturing depends greatly upon chemical and physical characteristics of raw materials, its quality and quantity, simplicity of its extraction and its costs. Ain El Sukhna area is surrounded by the Eocene limestone plateaus from the eastern and western sides. The plateaus are unconformity over the Permo-Triassic of Qiseib Formation that are made mainly of clays. The present study is aiming to preliminary assess the suitability and characterize raw materials (limestone and clay) in the western part of Zaafrana area for cement industry. Samples of limestone and clays were collected and analyzed for their main oxides. Geological and geochemical investigations indicated the suitability of the limestone of Sannor Formation and the Qiseib Formation kaolinitic clay for the cement industry in the area with considerable reserve. 


\section{Introduction}

Portland cement is a hydraulic powder material, which reacts with water to produce strength-bearing lattices. The mixture of aggregates, cement and water is concrete. The strength and durability of concrete makes it one of the most useful materials developed by man. In simple terms, cement is a manmade mineral structure created at high temperatures, mainly comprising lime $(\mathrm{CaO})$, Silica $\left(\mathrm{SiO}_{2}\right)$ and oxides of aluminum and iron $\left(\mathrm{Al}_{2} \mathrm{O}_{3}\right.$ and $\left.\mathrm{Fe}_{2} \mathrm{O}_{3}\right)$. The cement making process can be divided; first 'clinker' is made at temperatures of $1400^{\circ} \mathrm{C}$ (Fig.1), then the clinker is milled with other minerals to produce the powder we know as cement (Kosmatka et al., 2002).

The raw materials used to produce clinker include some naturally occurring minerals and some materials available as waste streams from other industries. The most common combination of ingredients is limestone (for calcium) coupled with much smaller quantities of clay, shale and sand (as a source of silica, aluminum and iron). Other 'alternative' raw materials such as mill scale, fly ash and slag are brought in from other industries. Most cement plants are normally located on limestone deposits and shale or clay reserves to mine this locally. Additives are usually brought in, such as sands, iron ore and/or mill scales. Raw materials used in Portland cement manufacturing must contain appropriate proportion of calcium oxides, silica, alumina and iron oxides. According to Hewlett and Peter (1997) and Taylor (1997), the optimum chemical composition of clinker would have $67 \% \mathrm{CaO}$, $22 \% \mathrm{SiO}_{2}, 5 \% \mathrm{Al}_{2} \mathrm{O}_{3}$ and $3 \% \mathrm{Fe}_{2} \mathrm{O}_{3}$. The relation between the four oxides has a direct impact on the clinker properties and its mineralogical compounds. Fortunately, different types of limestones and clays of different ages cover considerable parts of Egypt. In the North Eastern Portion of Egypt near the Red Sea coast, the carbonates usually form large extensive plateau that extending from Cairo to Red Sea coast.

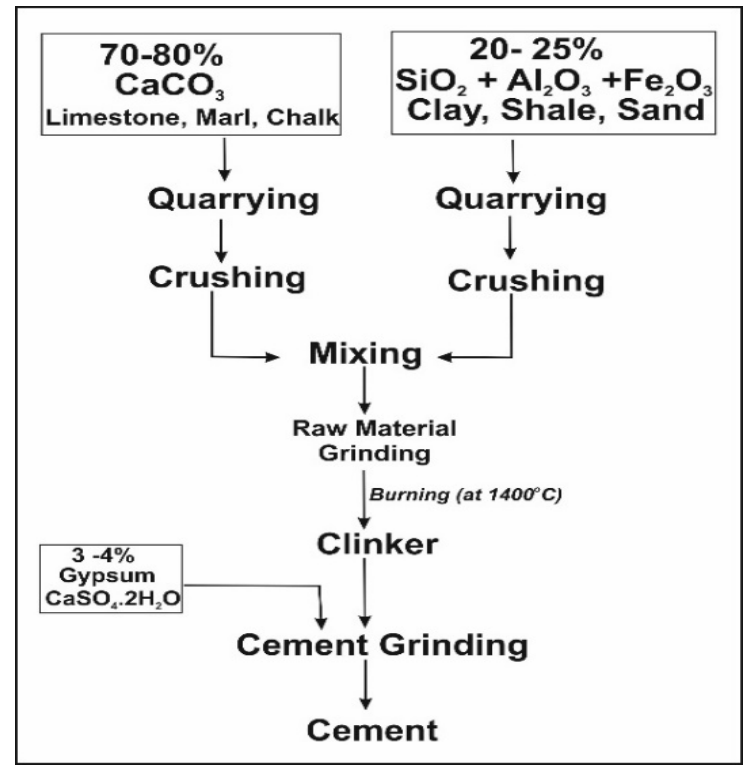

Fig.1: The life cycle of the cement production

Arabian Cement Company (ACC) plant is located at $29^{\circ} 47^{/} 43^{/ /} \mathrm{N}, 32^{\circ} 08^{/} 33^{/ /} \mathrm{E}$, north of El Sukhna old road. This plant is located at the foot slope of Gebel El Ramliya limestone (Fig.2). At Gebel El Ramliya, Middle Eocene Mokattam group consists of Sannor (Observatory) Formation shallow marine dense medium bedded limestone with local chert and numulites.

In the area east of El Sukhna, large extension of limestone forming the cap rock of the plateau exists on both sides of the Cairo-Suez Road. The plateau extends from east of EI Qattamiya to Red Sea. It extends to around 100 $\mathrm{km}$ east west and almost $200 \mathrm{~km}$ north east. The limestone plateau in the area is dissected by number of wadis. These wadis are; W. Gharaba, and W. Hagul. The existence of these wadis makes it possible for limestone quarrying and sampling due to the height of plateau and its steep slope (Fig.2).

Path from Reliance Heavy Industries (RHI) Clay Quarry (Abu Darag Clay Quarry) to Arabian Cement Company Plant is approximately $80 \mathrm{~km}$. The Red Kaolinitic Clay deposits are of economic significance in the Abu Darag area. These kaolinitic clay deposits are a part of the 
Paleozoic-Mesozoic sedimentary succession to the north-eastern part of Wadi Araba, along the southern slopes of the Northern Galala Plateau. The kaolinitic claystones of the Permo-Triassic (Qiseib Formation) are mostly sandy and alternate with sandstones in a cyclic pattern (Fig.
3). Clay in the area is overlain by an average of $3.5 \mathrm{~m}$ of sand and gravels (wadi deposits) and outcrops in few localities in the area before it covered by the reclaimed lands.

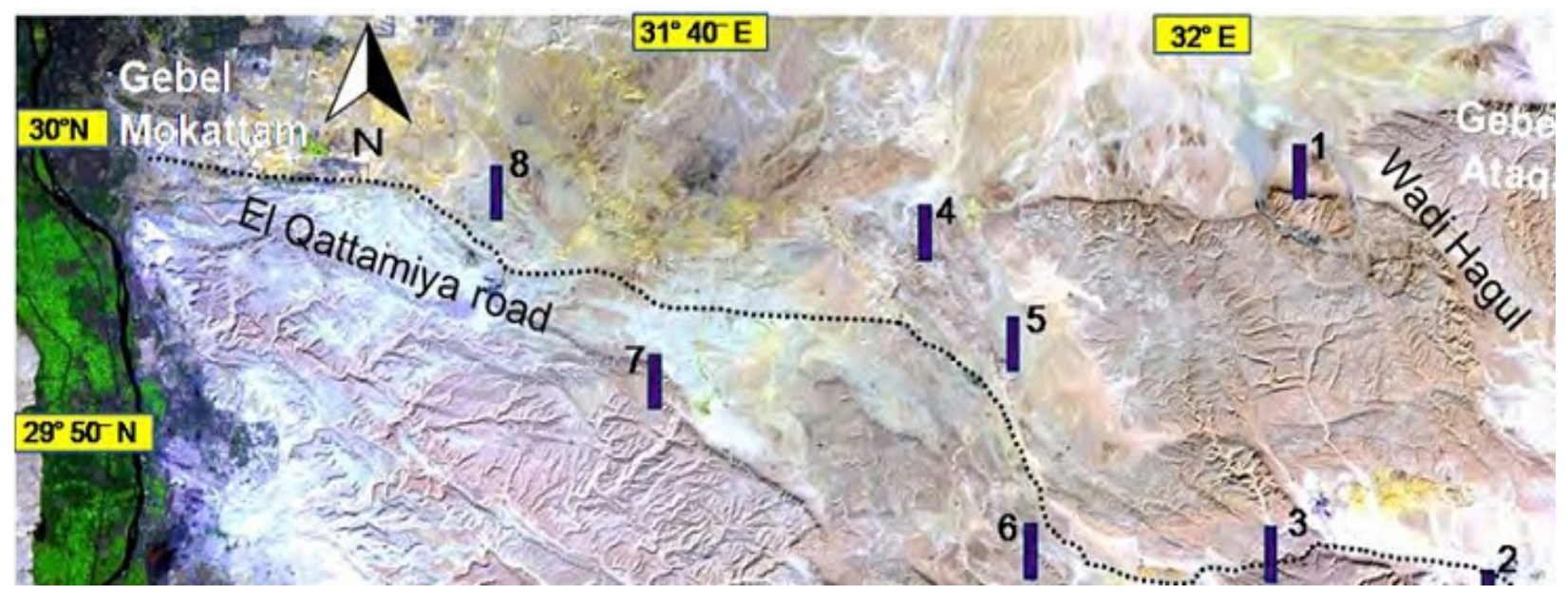

Fig. 2: ETM+ image of the study area showing the location of the studied sections:1 Gebel Abu Treifiya, 2 Gebel Akheider, 3 Gebel El Ramliya, 4 Gebel El Qattamiya, 5 Gebel Umm Reheiat, 6 Wadi Gharaba, 7 Gebel Abu Shama, and 8 Bir Gindaly.

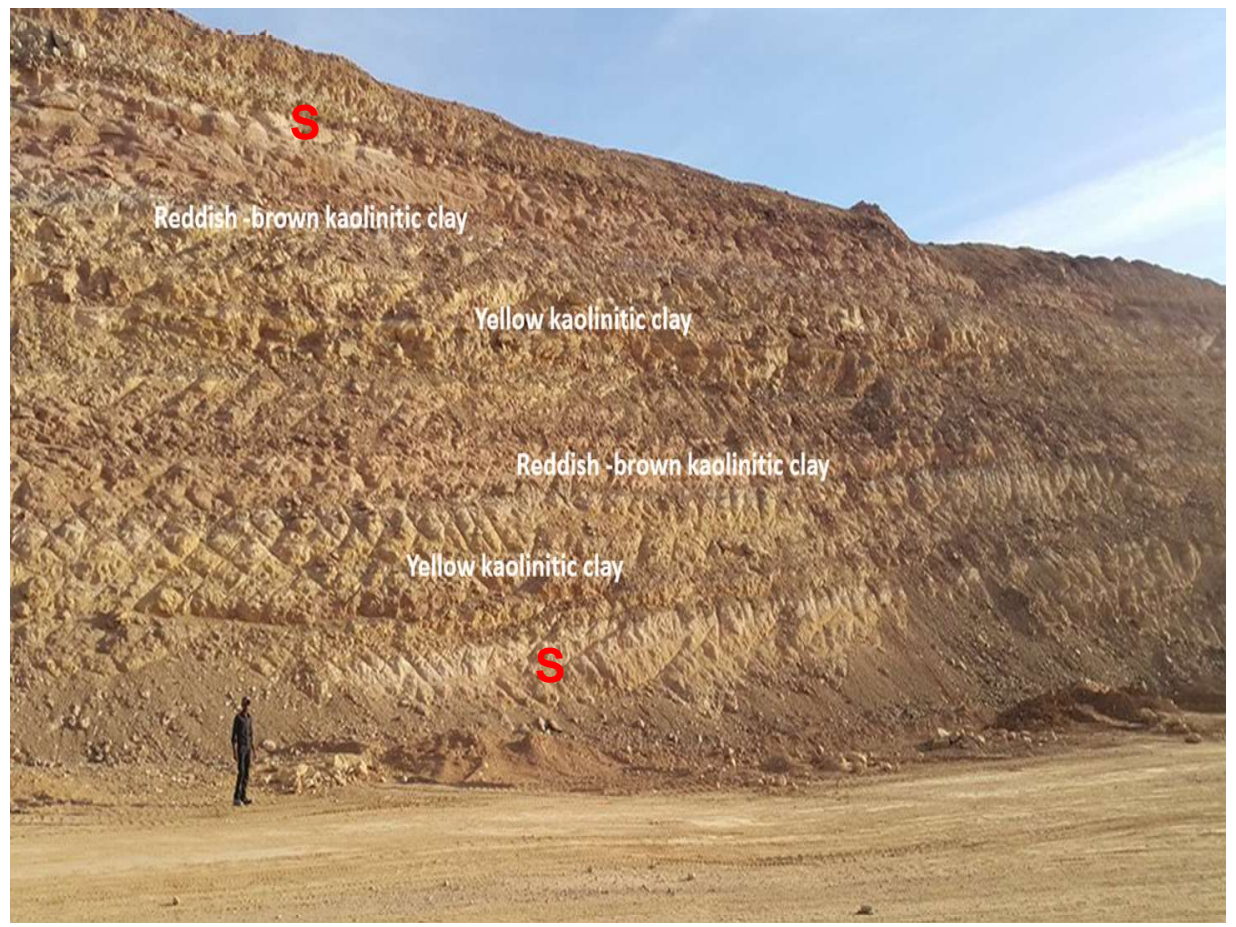

Fig.3: Intercalation flat- bedding variegated kaolinitic clay and sandstone in section II, Abu Darag Quarry, Red Sea area. Notice: upward gradation from thick to thin sandstone beds $(S)$ that occur between the kaolinitic clay; Lower horizon: Yellowish-white kaolinitic clay; and Upper horizon: Reddish-Brown kaolinitic clay. 
In June 2013 RHI (Reliance Heavy Industries) has signed a contract with the Egyptian Military - Al-Kheidma Al-Watania Sector. The contract is giving $\mathrm{RHI}$ the authority to extract the kaolinitic clays from an area of $1700 \mathrm{~m} \times 1000 \mathrm{~m}$ with minimum rate of supply of $50,000 \mathrm{~m}^{3}$ per month.

The clay deposit lies at the western coast of the Gulf of Suez. The clay occurrence is an almost flat lying sequence of clay, sandy clay, and sandstone that occupies the low lands south of Gabal El Galala El Baharia (Fig. 4).

The main valleys and tributaries that traversed the area had masked appreciable parts of the clay exposures.

In 2005 core drilling was performed for this area in 29 core holes (See figure 5) from the top surface layer to varying depth depending on the continuity of the clay succession in the subsurface. The sequence penetrated is an alternation of red kaolintic clay beds and sandy clay beds with frequent sandstone intercalations (Fig. 3). The red koalintic clay is massive, indurated, dense, and light to greyish- brown in colour. The clay of this quality is semi-consolidated, kaolinitic, detrital, fine grained and dry. The sand is poor to well sorted, ranging in size from very fine to coarse sand, and even fine pebble size. The colors of the clay and sand varieties vary depending on the iron oxide contents

These natural resources are not utilized for any forms of industries and therefore the aim of this research is to highlight the chemical composition of the existing resources (limestone and clay) and to investigate their suitability for cement industries and make it available for investors to develop the area for job creation and make use of such raw materials. Also the study concerns with the assessment of the raw materials reserve to guarantee the sustainability for manufacturing. Small-scale private quarries of clay and sand are exploited in a very smallscale for building stones.

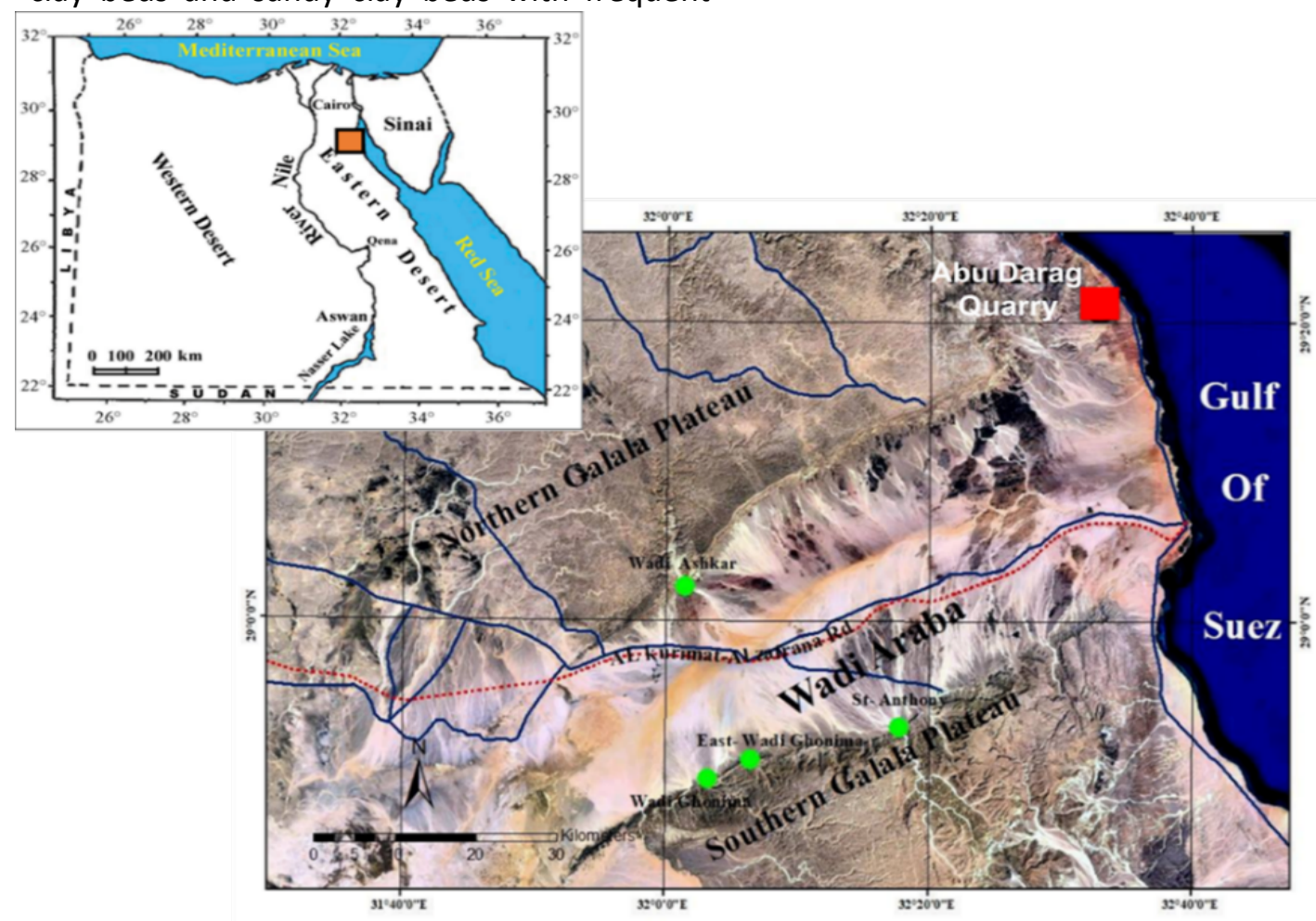

Fig.4: ETM+ image of the study area showing the location of the Wadi Araba area. Therectangular indicated to study Abu El Darag Clay Quarry area. 


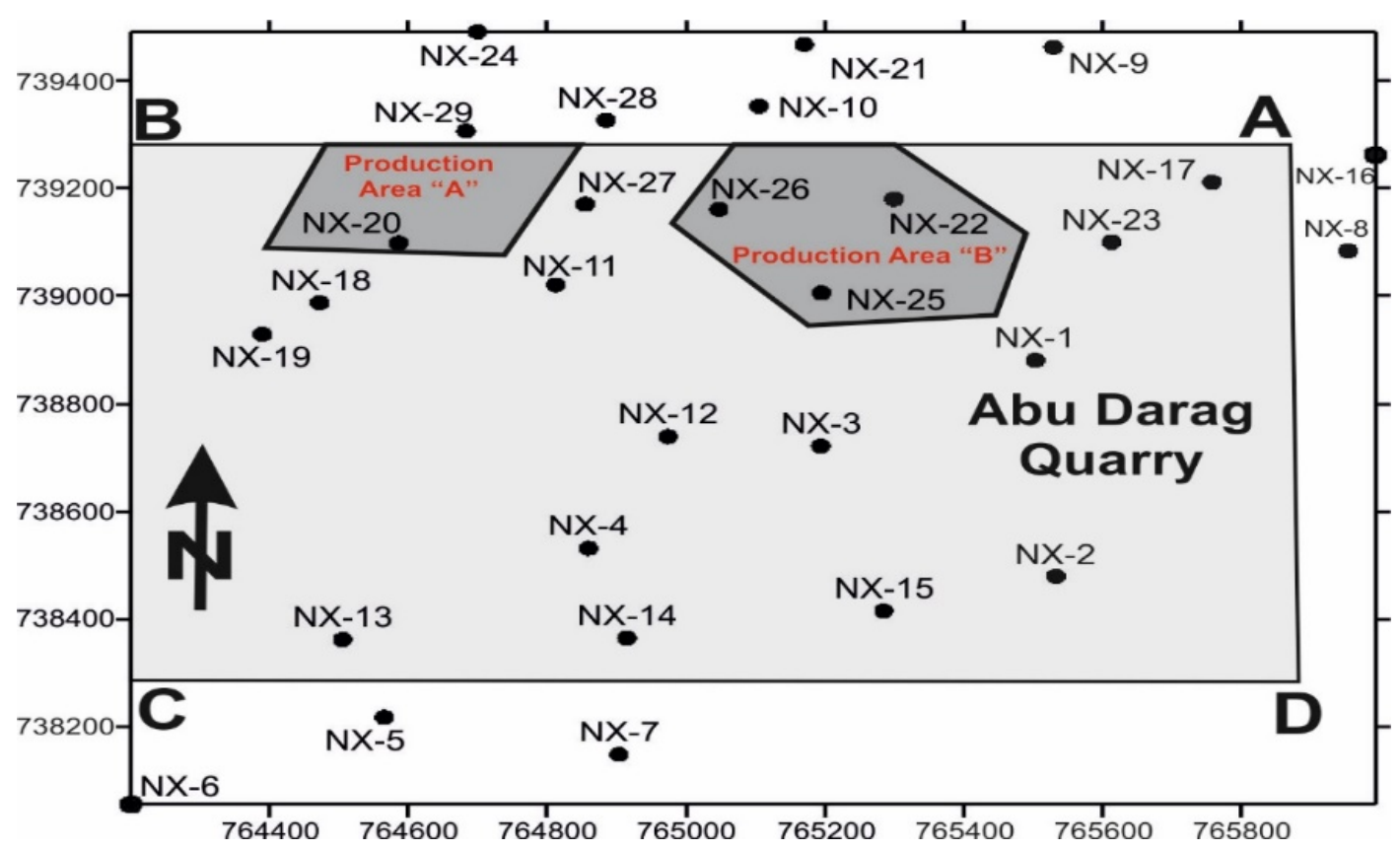

Fig.5: Areal distribution and location of 29 wells drilled in 2005 at Abu Darag area, Red Seaillustrations the Outlines boundaries of Abu Darag Quarry $(A B C D)$; as well as production Areas "A" and "B".
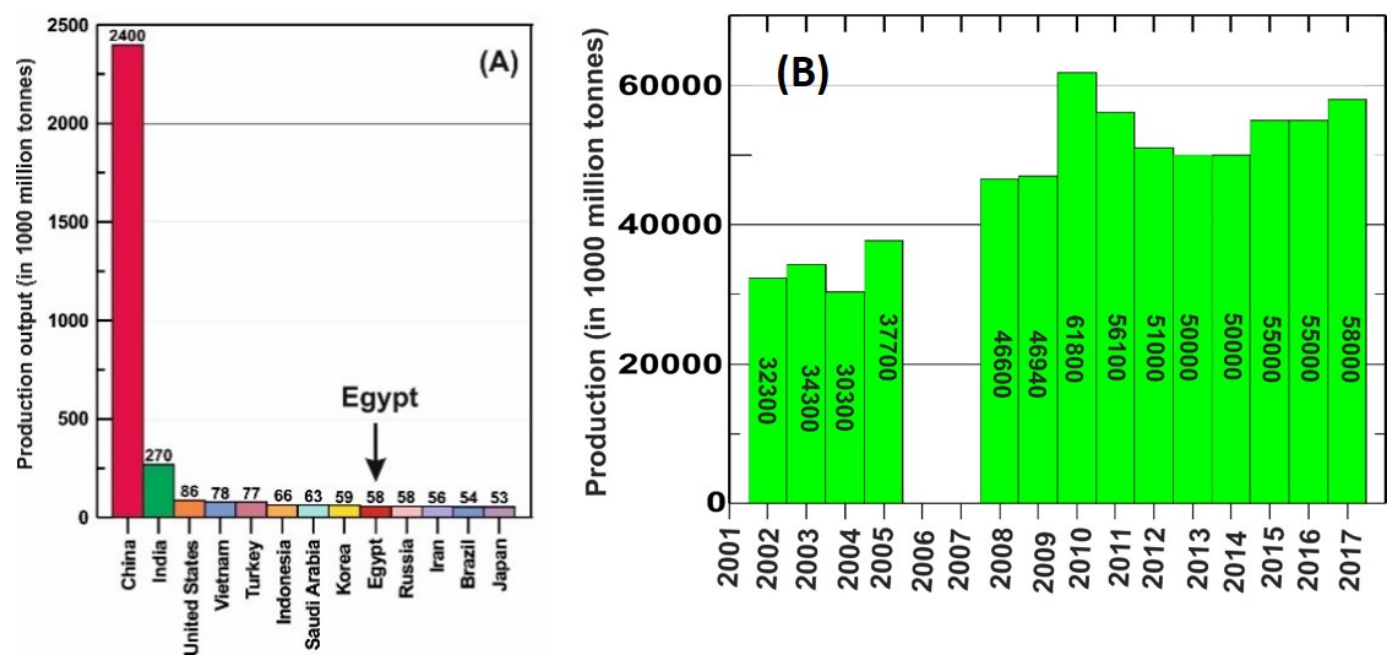

Fig.6: (A) Top Cement Producers in 2017; and (B) Egyptian cement production between 2001 and2017 (in 1000 million tonnes).

\section{International Cement Industry}

About 4,100,000 Mt (million metric tons) of cement were producing in 2017 by 13 countries (Fig.6A). Asian countries were the highest of global producer cement with $75 \%$ of the global productions by China; India; Vietnam; Korea, Indonesia; Turkey; Saudi Arabia; and Iran
(Fig.6A). China is the $1^{\text {st }}$ largest cement producer accounted for 2,400,000 Mt then India in the $2^{\text {nd }}$ position $(270,000 \mathrm{Mt})$. United States is the $3^{\text {rd }}$ largest cement producers ( 86300 million tons).

Egypt's cement production is estimated to be $1.4 \%(58,000 \mathrm{Mt})$ of the total world production 4,100,000 Mt (year 2017). On the other hand, Egypt is the largest cement producer 
in Africa and occupied $9^{\text {th }}$ position $(58,000 \mathrm{Mt}$, Fig.6B) as well as consumer in the world led by the enormous growth in the infrastructure and construction sector for the last two decades.

\section{Cement Manufacturing in Egypt}

The cement industry in Egypt started in the twentieth century. In 1927, the Tourah Portland Cement Company (TPCC) was established, followed by the Helwan Cement Company (HCC) south of Cairo in 1929. Later in 1948, Alexandria Cement Company (APCC) was established followed by National Cement Company (NCC) in 1956. In the 70s, the production capacities of the 4 cement companies reached around $4 \mathrm{Mt}$ (million metric tonnes) (ECS, 2009). The construction boom witnessed in the late 70s and 80s created high demand for cement that was met through imports because of the limited local production capacities, despite the opening of 3 new cement companies, Suez, Assuit and Amiryah, which started production throughout that period. In the mid-80s, Egypt became one of the largest cements importing countries in the world. During the 90s, 6 new cement companies were established to cope with the increasing construction activity and the resulting increasing cement demand, especially with the appearance of new sub-urban cities such as, AlSherouq, Al-Obour, $6^{\text {th }}$ of October, $10^{\text {th }}$ of Ramadan and Al-Sadat. Robust growth in the 1990s. Cement imports highest in 1999.

However, Egypt's cement net importing position prevailed during the 90 s and the early years of the $21^{\text {st }}$ century. Consequently, cement producers in Egypt increased their production capacities and enhanced their production lines to meet the surging local cement demand. In 2002, Egypt turned out to be a net exporter of cement and later in 2004 Egypt stopped importing cement and became one of the largest cement exporting countries in the region. Economic growth phase was slow during $2001 / 2002$. Cement consumption declined by $4 \%$ during the year with $10.8 \mathrm{Mt}$ of oversupply in 2003. Major national projects in Toshka, Suez Gulf and Sinai led to price war between producers trying to gain or defend existing market share. New entrants attracted to industry.

Since the establishment of the Tourah Portland Cement Company (TPCC) in 1927 the Egyptian cement industry (ECI) has witnessed a steady growth. Also, a future look at the developments shows that huge expansions are to be expected. By establishing new or expanding existing production lines or by establishing new cement companies during the next three years Egypt aims to cope with the increasing consumption of the local market. It is estimated that around $16 \mathrm{Mt}$ of clinker will be added, pushing the design capacity to $54.8 \mathrm{Mt}$ of clinker. As for cement, it is expected that about $18 \mathrm{Mt}$ will be added to the design capacity, which will increase from $46.6 \mathrm{Mt}$ in 2008 to around $61.8 \mathrm{Mt}$ in 2010. In 2017, the country's cement industry produced around 58 Mt of this building material. Furthermore, Egypt became one of the main exporting countries, with exports reaching their peak in 2017 at about $58 \mathrm{Mt}$ of cement (Fig.6A). This statistic represents the volume of cement that was produced in Egypt from 2008 through 2017 (Fig.6B). Currently, the installed capacity of cement industry in Egypt reached approximately $62 \mathrm{Mt}$ of cement per year, primarily from dry kilns, with only a small amount from seven wet kilns in two companies (Table 1; Fig.7). The satellite image in Fig.5 shows the locations of the 16 cement factories in Egypt.

Table 1 lists the production of each of the Egyptian cement-producing companies; all data refer to the year 2017, and in tandem with these data, the whole dataset has been updated with the latest figures by visiting a few Egyptian plants and doing an on-site survey due to the difficulty of acquiring statistics from a few plants because of breaching confidentiality in data acquisition. 
Table 1: Cement company's clinker production in Egypt.

\begin{tabular}{|c|c|c|c|c|c|}
\hline Group Name & $\begin{array}{l}\text { Company } \\
\text { Name }\end{array}$ & $\begin{array}{l}\text { Facility } \\
\text { Name }\end{array}$ & City & Klin Line* & $\begin{array}{l}\text { Clinker } \\
\text { Production } \\
\text { (Million } \\
\text { tonne) }\end{array}$ \\
\hline \multirow{7}{*}{$\begin{array}{l}\text { Italcementi } \\
\text { Group \{Suez } \\
\text { Cement } \\
\text { Group of } \\
\text { Companies } \\
(\text { SCGC)\} }\end{array}$} & $\begin{array}{l}\text { Helwan } \\
\text { Cement } \\
\text { Company (HCC) }\end{array}$ & Helwan & Helwan & $\begin{array}{l}\text { Dry Kiln } 1 \text { (plant 2), } \\
\text { Dry Kiln } 2 \text { (plant 2), } \\
\text { wet Kiln } 2 \text { (plant 1), } \\
\text { wet Kiln } 3 \text { (plant 1), } \\
\text { wet Kiln } 5 \text { (plant 1), } \\
\text { wet Kiln } 6 \text { (plant 1), } \\
\text { wet Kiln } 1 \text { (plant 3), } \\
\text { wet Kiln } 2 \text { (plant 3) }\end{array}$ & $4,009,340$ \\
\hline & $\begin{array}{l}\text { Tourah } \\
\text { Portland } \\
\text { Cement } \\
\text { Company } \\
\text { (TPCC) }\end{array}$ & Tourah & Tourah & $\begin{array}{l}\text { Kiln } 5 \text {, Kiln } 7 \text {, Kiln } 8 \text {, } \\
\text { Kiln } 9\end{array}$ & $2,474,412$ \\
\hline & $\begin{array}{l}\text { Suez Cement } \\
\text { Company } \\
\text { (QCP) }\end{array}$ & $\begin{array}{l}\text { Qattamya } \\
\text { Plant }\end{array}$ & Qattamya & Kiln 1 & $9,845,810$ \\
\hline & $\begin{array}{l}\text { Suez Cement } \\
\text { Company (SCC) }\end{array}$ & Suez Plant & Suez & Kiln 1, Kiln 2 & $2,100,710$ \\
\hline & $\begin{array}{l}\text { Suez Cement } \\
\text { Company }\end{array}$ & $\begin{array}{l}\text { El Minya } \\
\text { Plant SCC }\end{array}$ & El Minya & Kiln 1 & 287,666 \\
\hline & $\begin{array}{l}\text { National } \\
\text { Cement } \\
\text { Company (NCC) }\end{array}$ & Al-Tebbin & $\begin{array}{l}\text { South } \\
\text { Helwan }\end{array}$ & $\begin{array}{l}\text { Kiln } 1 \text { (wet), Kiln } 2 \\
\text { (wet), Kiln 3, Kiln } 4\end{array}$ & $3,031,951$ \\
\hline & $\begin{array}{l}\text { El Sewedy } \\
\text { Cement } \\
\text { Company } \\
\text { (ESCC) }\end{array}$ & $\begin{array}{l}\text { Ataqah- } \\
\text { Suez }\end{array}$ & Ataqah & Kiln 1 & \\
\hline $\begin{array}{l}\text { Lafarge } \\
\text { Holcim Ltd }\end{array}$ & $\begin{array}{l}\text { Lafarge } \\
\text { Cement Egypt, } \\
\text { S.A.E. (LCC) }\end{array}$ & Suez & Suez & $\begin{array}{l}\text { Kiln 1, Kiln 2, Kiln 3, } \\
\text { Kiln 4, Kiln } 5\end{array}$ & $8,295,478$ \\
\hline $\begin{array}{l}\text { Cementos La } \\
\text { Union, S.A. }\end{array}$ & $\begin{array}{l}\text { Arabian } \\
\text { Cement } \\
\text { Company (ACC) }\end{array}$ & $\begin{array}{l}\text { Ain El } \\
\text { Sukhuna }\end{array}$ & Suez & Kiln 1 & $2,030,428$ \\
\hline \multirow[t]{4}{*}{ Cemex } & $\begin{array}{ll}\text { Cemex Egypt } \\
\text { (Assiut } \\
\text { Cement) } \\
\end{array}$ & Assiut & Assiut & Kiln 1, Kiln 2, Kiln 3 & $4,706,112$ \\
\hline & $\begin{array}{l}\text { Wadi El Nile } \\
\text { Cement } \\
\text { Company } \\
\text { (WNCC) }\end{array}$ & $\begin{array}{l}\text { Wadi El } \\
\text { Nile } \\
\text { Cement } \\
\text { Company }\end{array}$ & $\begin{array}{ll}\text { Wadi } & \text { El } \\
\text { Ebyari/ } & \text { Al } \\
\text { Ashyab } & \end{array}$ & & \\
\hline & $\begin{array}{ll}\text { El } & \text { Naha } \\
\text { Cement } & \end{array}$ & Qena & Qena & & \\
\hline & $\begin{array}{l}\text { Medcom } \\
\text { Cement }\end{array}$ & $\begin{array}{l}\text { Aswan } \\
\text { Cement } \\
\text { Plant }\end{array}$ & Aswan & & \\
\hline
\end{tabular}




\begin{tabular}{|c|c|c|c|c|c|}
\hline & $\begin{array}{l}\text { Royal El Minya } \\
\text { Cement }\end{array}$ & El Minya & Samalut & & \\
\hline \multirow{2}{*}{ ASEC Cement } & \multirow{2}{*}{$\begin{array}{l}\text { Misr Cement } \\
\text { Qena Co. }\end{array}$} & $\begin{array}{l}\text { ASEC } \\
\text { Minya }\end{array}$ & Samalut & & \\
\hline & & Qena & Nasr City & Production line & $1,859.73$ \\
\hline $\begin{array}{l}\text { Helwan } \\
\text { Cement } \\
\text { Company }\end{array}$ & $\begin{array}{l}\text { Helwan }- \text { El } \\
\text { Menia White } \\
\text { Cement } \\
\text { Company }\end{array}$ & El Minya & El Minya & & \\
\hline \multirow{2}{*}{$\begin{array}{l}\text { Building } \\
\text { Materials } \\
\text { Industries } \\
\text { Company } \\
\text { (BMIC) }\end{array}$} & \multirow[b]{2}{*}{$\begin{array}{ll}\text { South } & \text { Valley } \\
\text { Cement } & \text { S.A.E. } \\
\text { (SVCC) } & \end{array}$} & Beni Suef & Beni Suef & Kiln 1 & $1,573,844$ \\
\hline & & $\begin{array}{l}\text { BMI } \\
\text { Assisut } \\
\text { Plant }\end{array}$ & Assiut & & \\
\hline \multirow[t]{2}{*}{$\begin{array}{l}\text { Egyptian } \\
\text { Armed Forces }\end{array}$} & \multirow[t]{2}{*}{$\begin{array}{l}\text { El Arish } \\
\text { Cement } \\
\text { Company }\end{array}$} & \multirow[t]{2}{*}{ El Arish } & Beni Suef & $\begin{array}{l}\text { Cement plant with six } \\
\text { production lines in } \\
\text { the Egyptian city of } \\
\text { Beni Suef }\end{array}$ & \\
\hline & & & El Arish & Kiln 1, Kiln 2 & \\
\hline \multirow[b]{2}{*}{ Titan Group } & $\begin{array}{ll}\text { Titan } & \text { Cement } \\
\text { Egypt } & \\
\end{array}$ & Beni Suef & Beni Suef & Kiln 1 & $1,573,844$ \\
\hline & $\begin{array}{l}\text { Alexandria } \\
\text { Portland } \\
\text { Cement } \\
\text { Company } \\
\text { (APCC) }\end{array}$ & El Mex & Alexandria & Kiln 1 & $1,500,005$ \\
\hline $\begin{array}{l}\text { Cimentos de } \\
\text { Portugal, } \\
\text { SGPS, S.A. } \\
\text { (Cimpor) }\end{array}$ & $\begin{array}{l}\text { Amreyah } \\
\text { Cement } \\
\text { Company }\end{array}$ & Amreyah & Burg al Arab & Kiln 1, Kiln 2 & $1,900,483$ \\
\hline $\begin{array}{l}\text { Aalborg } \\
\text { Portland A/S }\end{array}$ & $\begin{array}{l}\text { Sinai White } \\
\text { Portland } \\
\text { Cement Co. }\end{array}$ & $\begin{array}{l}\text { Sinai } \\
\text { White }\end{array}$ & El Arish & & \\
\hline Vicat Group & $\begin{array}{l}\text { Sinai Cement } \\
\text { Company }\end{array}$ & Sinai & El Arish & Kiln 1 & \\
\hline
\end{tabular}

* In terms of the kiln process type, primarily from dry kilns, with only a small amount from seven wet kilns in two companies (National cement and Helwan (Italcementi group). 


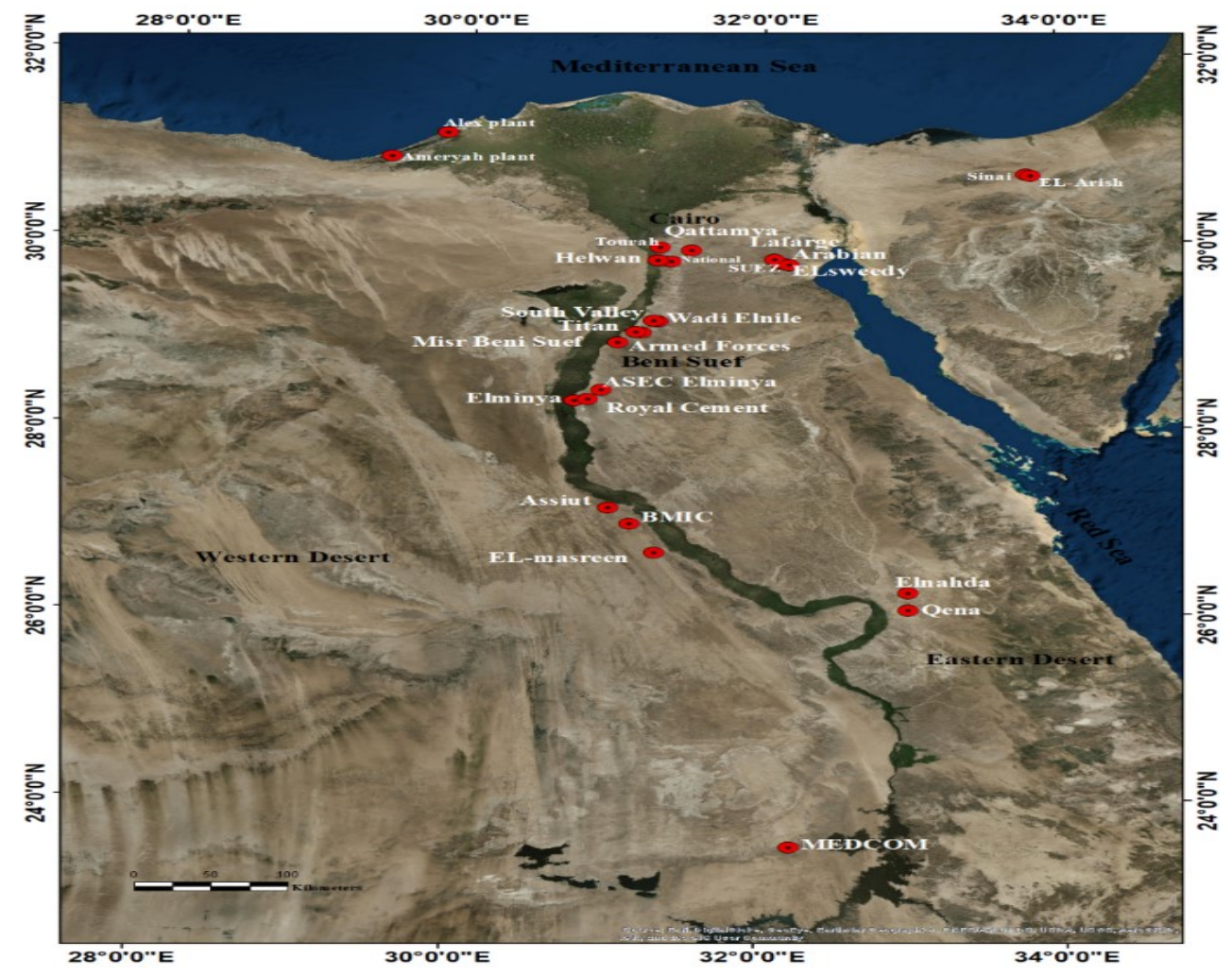

Fig.7: Distribution of cement factories in Egypt are highlighted with red indicators

\section{Raw Materials Sampling and Reserve Estimation}

According to the Egyptian Geological Survey reports, the thickness of limestone in the eastern side of Gebel Ataqa reaches $200 \mathrm{~m}$ or more. This thickness extends laterally for a long distance. This limestone becomes slightly dolomitic at the lower part and chalky at upper part. Arabian Cement Company (ACC) plant is located at $29^{\circ} 47^{\prime}$ $43^{/ /} \mathrm{N}, 32^{\circ} 08^{/} 33^{/ /} \mathrm{E}$, north of El Sukhna old road. This plant is located at the foot slope of Gebel El Ramliya limestone. At Gebel El Ramliya, Middle Eocene Mokattam group consisting of Sannor (Observatory) Formation shallow marine dense medium bedded limestone with local chert and numulites. Field observation and measurements indicate that the average thickness of the limestone can be quarried ranges between $45 \mathrm{~m}$ and $70 \mathrm{~m}$ (Sannor Formation). About 200 Million tonne of proven limestones can be quarrying for 50 years

Clays crop-out at several localities, mainly reddish-brown, grey to yellowish-grey, brown colour in parts and sandy in others. The thickness of these clays varies from place to another. In some places its thickness ranges from $5 \mathrm{~m}$ to $23 \mathrm{~m}$ of clay and silty clay with overburden reach to $3 \mathrm{~m}$ (EGSMA, 1988). Samples were collected from the study area for chemical analysis. Fifty-five samples of limestone in five areas in the southeastern part of the province were collected in the form of nine trenches. Twenty-two clay samples representing the Abu Darag areas were collected from quarries in the Abu Darag area (Fig.4). These samples were analyzed chemically by XRF and the physical properties were 
assessed. Table 2 shows the preferred range of some parameters in the raw materials for cement industries as given by Taylor (1997). Table 3 shows the chemical analysis of the 55 limestone samples collected from the Gabel El Ramlaya area, while the results of the chemical analysis of the 22 clay samples collected from Abu Darag area is given in Table 4. On the other hand, Table 5 illustrates the average chemical analysis of these analyzed samples of limestone and clay.

\section{Quality of the Raw Materials}

Portland cement is made by powder heating of batches mixture of limestone and clay and/or other materials up to a temperature of $\sim 1450^{\circ} \mathrm{C}$. Partial fusion occurs and nodules or balls of clinker are produced. The clinker is ground mixture with a $5 \%$ of raw gypsum to control rate of thin the produced cement. The cement clinker typically has a composition in the region of $67 \% \mathrm{CaO}, 22 \% \mathrm{SiO}_{2}, 5 \% \mathrm{Al}_{2} \mathrm{O}_{3}, 3 \%$ $\mathrm{Fe}_{2} \mathrm{O}_{3}$ as well as $3 \%$ of other components, and normally contains four major phases; alite, belite, Ca-aluminates and ferrite phases (Kosmatka et al., 2002). Other phases, such as alkali sulphates and calcium oxide are normally present in minor amounts (Taylor, 1964).

Cement experts are care for some factors that determine quality of the raw materials. The most important factors are the Lime Saturation Factor (LSF), the Silicate Ratio (SM) and the Aluminum Ratio (AM) (Cement Sustainability Production Report, 2011).

The LSF is used for proportioning the raw mix. It is the ratio of $\mathrm{CaO}$ to the other three main oxides and is calculated as: LSF = $\mathrm{CaO} /\left(2.8 \mathrm{SiO}_{2}+1.18 \mathrm{Al}_{2} \mathrm{O}_{3}+0.65 \mathrm{Fe}_{2} \mathrm{O}_{3}\right)$ and varies between 0.88 and 0.95 for Portland cement. Typical LSF values in modern clinkers are 0.92-0.98. Values above 1.0 indicate that free lime is likely to be present in the clinker. This is because, in principle, at LSF=1.0 all the free lime should have combined with belite $\left(2 \mathrm{CaO} . \mathrm{SiO}_{2}\right)$ to form alite $\left(3 \mathrm{CaO} \cdot \mathrm{SiO}_{2}\right)$. If the LSF is higher than 1.0, the surplus free lime has nothing with which to combine and will remain as free lime. In practice, the mixing of raw materials is never perfect and there are always regions within the clinker where the LSF is locally a little above, or a little below, the target for the clinker as a whole. This means that there is almost always some residual free lime, even where the LSF is considerably below 1.0. It also means that to convert virtually all the belite to alite, an LSF slightly above 1.0 is needed.

Table 2: Preferred range of some parameters in raw material (after Taylor, 1997).

\begin{tabular}{|c|c|c|c|}
\hline Item & Range (\%) & Preferred & Effects \\
\hline $\mathrm{K}_{2} \mathrm{O}, \mathrm{Na}_{2} \mathrm{O}$ & $0-1$ & $0.2-0.3$ & $\begin{array}{l}\text { Improve burnability at lower temperature and deteriorates } \\
\text { at higher temperature, creates operational problems }\end{array}$ \\
\hline $\mathrm{SO}_{3}{ }^{-2}$ & $0-4$ & $0.5-2$ & $\begin{array}{l}\text { When } \mathrm{S}>(\mathrm{Na}, \mathrm{K}) \text { acts as modifier of alkali recycle by forming } \\
\text { less volatile }(\mathrm{Na}, \mathrm{K}) \mathrm{SO}_{4} \text { compounds. } 2.5-4 \% \text { causes sulphate } \\
\text { expansion }\end{array}$ \\
\hline $\mathrm{Cl}^{-1}$ & $0-0.6$ & $0.0-0.015$ & $\begin{array}{l}\text { Increases ring formation, chloride in concrete promotes } \\
\text { corrosion of steel }\end{array}$ \\
\hline MgO & $0-5$ & $0-2$ & $\begin{array}{l}\text { Reduce viscosity of liquid phase, quicker formation of } C_{3} S \\
\text { then decomposition into } C_{2} S\end{array}$ \\
\hline LSF & 88-95 & & \\
\hline SM & $>3$ & & \\
\hline AM & $>2$ & $1.6-1.8$ & \\
\hline
\end{tabular}


Table 3: Chemical analysis of limestone from Sannor Formation at Gebel El Ramliya

\begin{tabular}{|c|c|c|c|c|c|c|c|c|c|c|c|c|c|c|c|}
\hline Time & SmpGrp & $\mathrm{SiO}_{2}$ & $\mathrm{Al}_{2} \mathrm{O}_{3}$ & $\mathrm{Fe}_{2} \mathrm{O}_{3}$ & MgO & $\mathrm{CaO}$ & $\mathrm{Na}_{2} \mathrm{O}$ & $\mathrm{K}_{2} \mathrm{O}$ & $\mathrm{SO}_{3}$ & $\mathrm{Cl}$ & LOI & $\mathrm{CaCO}_{3}$ & LSF & SM & AM \\
\hline $5 / 1 / 20180: 00$ & Crush_Lst & 0.38 & 0.12 & 0.05 & 0.86 & 53.74 & 0.09 & 0.01 & 0.30 & 0.03 & 43.18 & 95.13 & 4341 & 2.24 & 2.40 \\
\hline 5/1/2018 18:51 & LSLOADING & 0.70 & 0.28 & 0.22 & 0.49 & 51.95 & 0.08 & 0.02 & 0.24 & 0.04 & 41.36 & 91.96 & 2135 & 1.40 & 1.27 \\
\hline 5/1/2018 18:51 & LSLOADING & 0.15 & 0.09 & 0.04 & 0.59 & 54.00 & 0.09 & 0.01 & 0.06 & 0.05 & 43.09 & 95.59 & 9779 & 1.15 & 2.25 \\
\hline $5 / 2 / 20180: 00$ & Crush_Lst & 0.16 & 0.09 & 0.04 & 0.92 & 54.44 & 0.07 & 0.01 & 0.09 & 0.02 & 43.79 & 96.37 & 9383 & 1.23 & 2.25 \\
\hline $5 / 3 / 201812: 00$ & Crush_Lst & 0.53 & 0.31 & 0.15 & 0.37 & 52.91 & 0.06 & 0.01 & 0.05 & 0.02 & 41.98 & 93.66 & 2717 & 1.15 & 2.07 \\
\hline $5 / 3 / 2018$ 13:30 & LSLOADING & 0.53 & 0.31 & 0.15 & 0.36 & 52.92 & 0.06 & 0.01 & 0.05 & 0.02 & 41.99 & 93.68 & 2718 & 1.15 & 2.07 \\
\hline 5/4/2018 0:00 & Crush_Lst & 2.11 & 1.05 & 0.59 & 0.70 & 47.48 & 0.15 & 0.04 & 0.07 & 0.07 & 38.08 & 84.05 & 631 & 1.29 & 1.78 \\
\hline $5 / 5 / 201812: 00$ & Crush_Lst & 0.62 & 0.17 & 0.07 & 1.04 & 52.17 & 0.17 & 0.05 & 0.10 & 0.09 & 42.12 & 92.35 & 2632 & 2.58 & 2.43 \\
\hline $5 / 6 / 20180: 00$ & Crush_Lst & 0.35 & 0.19 & 0.52 & 0.54 & 54.35 & 0.08 & 0.02 & 0.13 & 0.04 & 43.29 & 96.21 & 3524 & 0.49 & 0.37 \\
\hline $5 / 6 / 2018$ 12:24 & LSLOADING & 0.07 & 0.06 & 0.03 & 0.20 & 56.00 & 0.05 & 0.01 & 0.07 & 0.02 & 44.24 & 99.13 & 19560 & 0.78 & 2.00 \\
\hline $5 / 6 / 2018$ 12:24 & LSLOADING & 0.09 & 0.06 & 0.03 & 2.69 & 51.41 & 0.11 & 0.01 & 0.06 & 0.06 & 43.34 & 91.00 & 15019 & 1.00 & 2.00 \\
\hline 5/7/2018 0:00 & Crush_Lst & 0.05 & 0.06 & 0.06 & 7.67 & 43.04 & 0.24 & 0.01 & 0.12 & 0.09 & 42.17 & 76.19 & 17230 & 0.42 & 1.00 \\
\hline $5 / 7 / 201812: 00$ & Crush_Lst & 0.21 & 0.10 & 0.05 & 0.21 & 55.45 & 0.06 & 0.02 & 0.11 & 0.02 & 43.81 & 98.16 & 7508 & 1.40 & 2.00 \\
\hline $5 / 7 / 2018$ 12:29 & LSLOADING & 0.20 & 0.10 & 0.05 & 0.20 & 55.47 & 0.06 & 0.02 & 0.11 & 0.02 & 43.81 & 98.19 & 7807 & 1.33 & 2.00 \\
\hline $5 / 7 / 2018$ 12:29 & LSLOADING & 0.06 & 0.06 & 0.02 & 0.62 & 55.38 & 0.06 & 0.01 & 0.09 & 0.01 & 44.21 & 98.03 & 21994 & 0.75 & 3.00 \\
\hline $5 / 7 / 2018$ 12:29 & LSLOADING & 0.27 & 0.09 & 0.06 & 0.46 & 54.70 & 0.07 & 0.02 & 0.11 & 0.04 & 43.49 & 96.83 & 6070 & 1.80 & 1.50 \\
\hline $5 / 7 / 2018$ 12:29 & LSLOADING & 0.06 & 0.05 & 0.08 & 8.18 & 43.07 & 0.20 & 0.01 & 0.10 & 0.08 & 42.76 & 76.24 & 15437 & 0.46 & 0.63 \\
\hline $5 / 8 / 20180: 00$ & Crush_Lst & 1.03 & 0.47 & 0.28 & 0.13 & 52.36 & 0.07 & 0.02 & 0.10 & 0.02 & 41.30 & 92.69 & 1446 & 1.37 & 1.68 \\
\hline 5/8/2018 14:15 & LSLOADING & 0.11 & 0.06 & 0.05 & 1.25 & 53.30 & 0.08 & 0.01 & 0.06 & 0.04 & 43.26 & 94.35 & 12959 & 1.00 & 1.20 \\
\hline 5/8/2018 14:16 & LSLOADING & 0.50 & 0.15 & 0.11 & 2.87 & 49.00 & 0.15 & 0.02 & 0.08 & 0.10 & 41.63 & 86.74 & 2972 & 1.92 & 1.36 \\
\hline $5 / 10 / 20180: 00$ & Crush_Lst & 0.53 & 0.21 & 0.07 & 1.41 & 52.40 & 0.14 & 0.02 & 0.05 & 0.12 & 42.73 & 92.76 & 2948 & 1.89 & 3.00 \\
\hline $5 / 11 / 201812: 00$ & Crush_Lst & 1.44 & 0.37 & 0.24 & 0.16 & 50.80 & 0.15 & 0.08 & 0.14 & 0.03 & 40.10 & 89.92 & 1098 & 2.36 & 1.54 \\
\hline 5/12/2018 17:41 & LSLOADING & 0.54 & 0.29 & 0.18 & 0.36 & 53.07 & 0.05 & 0.02 & 0.06 & 0.02 & 42.10 & 93.94 & 2692 & 1.15 & 1.61 \\
\hline $5 / 12 / 2018$ 17:41 & LSLOADING & 0.10 & 0.09 & 0.05 & 0.80 & 54.13 & 0.05 & 0.01 & 0.08 & 0.01 & 43.42 & 95.82 & 12928 & 0.71 & 1.80 \\
\hline $5 / 13 / 20180: 00$ & Crush_Lst & 0.08 & 0.07 & 0.03 & 1.27 & 53.93 & 0.11 & 0.01 & 0.05 & 0.08 & 43.75 & 95.46 & 16538 & 0.80 & 2.33 \\
\hline $5 / 14 / 2018$ 13:24 & LSLOADING & 0.39 & 0.14 & 0.08 & 0.15 & 54.51 & 0.08 & 0.02 & 0.08 & 0.02 & 43.00 & 96.49 & 4164 & 1.77 & 1.75 \\
\hline $5 / 14 / 201813: 24$ & LSLOADING & 0.22 & 0.11 & 0.05 & 0.40 & 54.26 & 0.07 & 0.02 & 0.08 & 0.02 & 43.09 & 96.05 & 6972 & 1.38 & 2.20 \\
\hline 5/14/2018 13:24 & LSLOADING & 0.10 & 0.07 & 0.03 & 0.13 & 55.25 & 0.05 & 0.01 & 0.06 & 0.01 & 43.57 & 97.80 & 14460 & 1.00 & 2.33 \\
\hline
\end{tabular}


Table 3 (Cont.)

\begin{tabular}{|c|c|c|c|c|c|c|c|c|c|c|c|c|c|c|c|}
\hline Time & SmpGrp & $\mathrm{SiO}_{2}$ & $\mathrm{Al}_{2} \mathrm{O}_{3}$ & $\mathrm{Fe}_{2} \mathrm{O}_{3}$ & MgO & $\mathrm{CaO}$ & $\mathrm{Na}_{2} \mathrm{O}$ & $\mathrm{K}_{2} \mathrm{O}$ & $\mathrm{SO}_{3}$ & $\mathrm{Cl}$ & LOI & $\mathrm{CaCO}_{3}$ & LSF & SM & AM \\
\hline $5 / 16 / 2018$ 12:00 & Crush_Lst & 0.18 & 0.09 & 0.04 & 1.68 & 52.26 & 0.05 & 0.01 & 0.17 & 0.01 & 42.91 & 92.51 & 8214 & 1.38 & 2.25 \\
\hline $5 / 16 / 201814: 33$ & LSLOADING & 0.17 & 0.08 & 0.04 & 1.68 & 52.26 & 0.05 & 0.01 & 0.17 & 0.01 & 42.91 & 92.51 & 8763 & 1.42 & 2.00 \\
\hline $5 / 17 / 20180: 00$ & Crush_Lst & 0.19 & 0.10 & 0.05 & 1.69 & 52.79 & 0.10 & 0.01 & 0.15 & 0.06 & 43.34 & 93.45 & 7735 & 1.27 & 2.00 \\
\hline $5 / 17 / 2018$ 13:23 & LSLOADING & 0.52 & 0.29 & 0.19 & 0.15 & 53.69 & 0.03 & 0.02 & 0.03 & 0.01 & 42.37 & 95.04 & 2794 & 1.08 & 1.53 \\
\hline 5/17/2018 13:24 & LSLOADING & 0.10 & 0.09 & 0.03 & 0.08 & 55.56 & 0.03 & 0.01 & 0.03 & 0.00 & 43.76 & 98.35 & 13695 & 0.83 & 3.00 \\
\hline $5 / 17 / 2018$ 13:24 & LSLOADING & 0.40 & 0.12 & 0.09 & 0.12 & 54.21 & 0.05 & 0.02 & 0.15 & 0.03 & 42.74 & 95.96 & 4107 & 1.90 & 1.33 \\
\hline $5 / 18 / 20180: 00$ & Crush_Lst & 0.36 & 0.13 & 0.06 & 2.76 & 49.81 & 0.14 & 0.02 & 0.48 & 0.08 & 42.16 & 88.17 & 4149 & 1.89 & 2.17 \\
\hline $5 / 18 / 2018$ 12:00 & Crush_Lst & 0.30 & 0.12 & 0.06 & 0.69 & 53.00 & 0.04 & 0.02 & 0.14 & 0.01 & 42.39 & 93.82 & 5193 & 1.67 & 2.00 \\
\hline 5/19/2018 0:00 & Crush_Lst & 0.13 & 0.08 & 0.03 & 0.35 & 55.51 & 0.06 & 0.01 & 0.10 & 0.02 & 44.01 & 98.26 & 11615 & 1.18 & 2.67 \\
\hline $5 / 19 / 201812: 00$ & Crush_Lst & 0.10 & 0.07 & 0.05 & 1.47 & 53.76 & 0.05 & 0.01 & 0.19 & 0.02 & 43.83 & 95.16 & 13607 & 0.83 & 1.40 \\
\hline $5 / 20 / 201812: 00$ & Crush_Lst & 0.42 & 0.14 & 0.10 & 0.15 & 54.41 & 0.06 & 0.02 & 0.36 & 0.03 & 42.94 & 96.31 & 3869 & 1.75 & 1.40 \\
\hline $5 / 20 / 201814: 37$ & LSLOADING & 0.36 & 0.17 & 0.09 & 0.42 & 54.67 & 0.05 & 0.02 & 0.08 & 0.01 & 43.43 & 96.77 & 4315 & 1.38 & 1.89 \\
\hline $5 / 20 / 201814: 37$ & LSLOADING & 0.42 & 0.14 & 0.10 & 0.15 & 54.38 & 0.07 & 0.02 & 0.35 & 0.03 & 42.91 & 96.26 & 3867 & 1.75 & 1.40 \\
\hline $5 / 22 / 201814: 31$ & LSLOADING & 0.23 & 0.11 & 0.09 & 0.94 & 54.13 & 0.05 & 0.01 & 0.12 & 0.02 & 43.57 & 95.82 & 6504 & 1.15 & 1.22 \\
\hline $5 / 22 / 201814: 31$ & LSLOADING & 0.20 & 0.09 & 0.09 & 1.04 & 53.88 & 0.05 & 0.01 & 0.07 & 0.03 & 43.48 & 95.38 & 7435 & 1.11 & 1.00 \\
\hline $5 / 22 / 201814: 31$ & LSLOADING & 0.20 & 0.09 & 0.08 & 0.37 & 55.25 & 0.05 & 0.01 & 0.09 & 0.02 & 43.83 & 97.80 & 7693 & 1.18 & 1.13 \\
\hline $5 / 23 / 20180: 00$ & Crush_Lst & 0.20 & 0.07 & 0.06 & 0.46 & 55.13 & 0.04 & 0.01 & 0.12 & 0.02 & 43.83 & 97.59 & 8088 & 1.54 & 1.17 \\
\hline $5 / 24 / 201812: 00$ & Crush_Lst & 0.27 & 0.10 & 0.06 & 0.67 & 54.34 & 0.07 & 0.02 & 0.10 & 0.03 & 43.44 & 96.19 & 5952 & 1.69 & 1.67 \\
\hline $5 / 25 / 20180: 00$ & Crush_Lst & 0.22 & 0.09 & 0.05 & 0.95 & 54.14 & 0.08 & 0.01 & 0.04 & 0.06 & 43.58 & 95.84 & 7174 & 1.57 & 1.80 \\
\hline $5 / 26 / 20180: 00$ & Crush_Lst & 0.85 & 0.30 & 0.22 & 1.20 & 50.65 & 0.09 & 0.03 & 0.53 & 0.05 & 41.11 & 89.66 & 1761 & 1.63 & 1.36 \\
\hline $5 / 27 / 20180: 00$ & Crush_Lst & 1.32 & 0.47 & 0.28 & 0.64 & 50.81 & 0.08 & 0.03 & 0.07 & 0.01 & 40.65 & 89.94 & 1146 & 1.76 & 1.68 \\
\hline $5 / 27 / 201812: 00$ & Crush_Lst & 0.31 & 0.10 & 0.08 & 0.96 & 52.95 & 0.07 & 0.02 & 0.13 & 0.02 & 42.66 & 93.73 & 5101 & 1.72 & 1.25 \\
\hline $5 / 27 / 2018$ 12:37 & LSLOADING & 0.09 & 0.06 & 0.04 & 1.01 & 53.54 & 0.05 & 0.01 & 0.13 & 0.01 & 43.18 & 94.77 & 15350 & 0.90 & 1.50 \\
\hline $5 / 27 / 2018$ 12:37 & LSLOADING & 0.30 & 0.10 & 0.08 & 0.96 & 52.94 & 0.07 & 0.02 & 0.13 & 0.02 & 42.65 & 93.71 & 5242 & 1.67 & 1.25 \\
\hline $5 / 29 / 2018$ 15:42 & LSLOADING & 0.31 & 0.13 & 0.10 & 1.26 & 52.52 & 0.09 & 0.03 & 0.05 & 0.02 & 42.65 & 92.97 & 4834 & 1.35 & 1.30 \\
\hline $5 / 29 / 2018$ 15:42 & LSLOADING & 1.58 & 0.47 & 0.18 & 1.19 & 48.46 & 0.23 & 0.11 & 0.10 & 0.03 & 39.38 & 85.78 & 951 & 2.43 & 2.61 \\
\hline $5 / 30 / 20180: 00$ & Crush_Lst & 0.22 & 0.09 & 0.06 & 0.67 & 54.21 & 0.06 & 0.02 & 0.24 & 0.03 & 43.34 & 95.96 & 7122 & 1.47 & 1.50 \\
\hline
\end{tabular}

Lime Saturation Factor $(\mathrm{LSF})=\mathrm{CaO} /\left(2.8 \mathrm{SiO}_{2}+1.18 \mathrm{Al}_{2} \mathrm{O}_{3}+0.65 \mathrm{Fe}_{2} \mathrm{O}_{3}\right)$

Silicate Ratio (SM) $=\mathrm{SiO}_{2} /\left(\mathrm{Al}_{2} \mathrm{O}_{3}+\mathrm{Fe}_{2} \mathrm{O}_{3}\right)$.

Aluminum Ratio $(\mathrm{AM})=\left(\mathrm{Al}_{2} \mathrm{O}_{3} /\left(\mathrm{Fe}_{2} \mathrm{O}_{3}\right)\right.$ 
Table 4: Chemical analysis of kaolinitic clay from Abu Darag area.

\begin{tabular}{|c|c|c|c|c|c|c|c|c|c|c|c|c|c|}
\hline ate & $\mathrm{SiO}_{2}$ & $\mathrm{Al}_{2} \mathrm{O}_{3}$ & $\mathrm{Fe}_{2} \mathrm{O}_{3}$ & MgO & $\mathrm{CaO}$ & $\mathrm{Na}_{2} \mathrm{O}$ & $\mathrm{K}_{2} \mathrm{O}$ & $\mathrm{SO}_{3}$ & $\mathrm{Cl}$ & LOI & LSF & SM & AM \\
\hline Tue & 36 & 14.14 & 2 & 27 & 2 & & & 70 & & & 6 & .26 & .82 \\
\hline Wednesday, May 2, 2018 & 60.01 & 17.78 & 7.50 & 0.35 & 0.31 & 0.17 & 0.67 & 0.09 & 0.19 & 12.44 & 0.16 & 2.37 & 2.37 \\
\hline Thursday, May 3, 2018 & 59.34 & 17.65 & 7.73 & 0.23 & 0.31 & 0.16 & 0.60 & 0.19 & 0.18 & 13.12 & 0.16 & 2.34 & 2.28 \\
\hline Friday, May 4, 2018 & 60.07 & 16.50 & 7.83 & 0.31 & 0.31 & 0.13 & 0.51 & 0.19 & 0.17 & 13.49 & 0.16 & 2.47 & 2.11 \\
\hline Saturday, May 5, 2018 & 61.02 & 16.61 & 6.47 & 0.23 & 0.31 & 0.15 & 0.54 & 0.21 & 0.18 & 13.76 & 0.16 & 2.64 & 2.57 \\
\hline Sunday, May 6, 2018 & 62.85 & 15.59 & 6.77 & 0.20 & 0.31 & 0.23 & 0.74 & 0.13 & 0.10 & 12.62 & 0.15 & 2.81 & 2.30 \\
\hline Monday, May 7, 2018 & 62.24 & 15.78 & 7.22 & 0.25 & 0.30 & 0.13 & 0.64 & 0.04 & 0.14 & 12.60 & 0.15 & 2.71 & 2.19 \\
\hline Tuesday, May 8, 2018 & 64.34 & 13.53 & 7.45 & 0.17 & 0.31 & 0.11 & 0.58 & 0.11 & 0.07 & 12.88 & 0.15 & 3.07 & 1.82 \\
\hline Wednesday, May 9, 2018 & 64.20 & 14.23 & 6.67 & 0.32 & 0.31 & 0.15 & 0.68 & 0.06 & 0.06 & 12.87 & 0.15 & 3.07 & 2.13 \\
\hline Thursday, May 10, 2018 & 63.27 & 15.45 & 7.23 & 0.16 & 0.30 & 0.20 & 0.60 & 0.04 & 0.06 & 12.26 & 0.15 & 2.79 & 2.14 \\
\hline Friday, May 11, 2018 & 64.27 & 14.00 & 6.50 & 0.19 & 0.30 & 0.16 & 0.76 & 0.48 & 0.17 & 12.75 & 0.15 & 3.14 & 2.15 \\
\hline Saturday, May 12, 2018 & 61.54 & 14.92 & 7.67 & 0.18 & 0.31 & 0.12 & 0.62 & 0.07 & 0.14 & 13.95 & 0.16 & 2.72 & 1.94 \\
\hline Sunday, May 13, 2018 & 60.49 & 15.45 & 628 & 0.26 & 0.31 & 0.14 & 0.59 & 0.31 & 0.14 & 15.54 & 0.16 & 2.78 & 2.46 \\
\hline Monday, May 14, 2018 & 64.29 & 12.84 & 5.93 & 0.28 & 0.31 & 0.11 & 0.46 & 0.13 & 0.12 & 15.05 & 0.16 & 3.43 & 2.17 \\
\hline Tuesday, May 15, 2018 & 60.03 & 16.96 & 8.58 & 0.27 & 0.31 & 0.18 & 0.50 & 0.05 & 0.10 & 12.51 & 0.16 & 2.35 & 1.98 \\
\hline Wednesday, May 16, 2018 & 61.03 & 17.60 & 8.09 & 0.22 & 0.30 & 0.17 & 0.66 & 0.02 & 0.11 & 11.30 & 0.15 & 2.37 & 2.17 \\
\hline Friday, May 18, 2018 & 60.30 & 18.34 & 6.80 & 0.21 & 0.30 & 0.12 & 0.44 & 0.04 & 0.09 & 12.92 & 0.15 & 2.40 & 2.70 \\
\hline Saturday, May 19, 2018 & 63.78 & 14.74 & 5.73 & 0.26 & 0.31 & 0.13 & 0.60 & 0.43 & 0.10 & 13.43 & 0.15 & 3.12 & 2.57 \\
\hline Sunday, May 20, 2018 & 62.60 & 17.35 & 5.97 & 0.23 & 0.31 & 0.18 & 0.61 & 0.12 & 0.09 & 12.05 & 0.15 & 2.68 & 2.90 \\
\hline Monday, May 21, 2018 & 61.53 & 19.89 & 6.99 & 0.28 & 0.31 & 0.10 & 0.63 & 0.21 & 0.10 & 9.42 & 0.15 & 2.29 & 2.84 \\
\hline Tuesday, May 22, 2018 & 63.16 & 19.75 & 6.47 & 0.22 & 0.31 & 0.22 & 0.56 & 0.12 & 0.09 & 8.64 & 0.15 & 2.41 & 3.05 \\
\hline Sunday, May 27, 2018 & 61.53 & 19.89 & 6.99 & 0.28 & 0.31 & 0.10 & 0.63 & 0.21 & 0.10 & 9.42 & 0.15 & 2.29 & 2.84 \\
\hline Monday, May 28, 2018 & 63.16 & 19.75 & 6.47 & 0.22 & 0.31 & 0.22 & 0.56 & 0.12 & 0.09 & 8.64 & 0.15 & 2.41 & 3.05 \\
\hline Tuesday, May 29, 2018 & 57.14 & 22.97 & 9.25 & 0.22 & 0.30 & 0.12 & 0.60 & 0.02 & 0.05 & 8.85 & 0.16 & 1.77 & 2.48 \\
\hline Wednesday, May 30, 2018 & 57.07 & 22.91 & 7.93 & 0.23 & 0.31 & 0.10 & 0.57 & 0.02 & 0.06 & 10.31 & 0.16 & 1.85 & 2.89 \\
\hline Thursday, May 31, 2018 & 59.90 & 21.40 & 8.25 & 0.22 & 0.30 & 0.15 & 0.58 & 0.05 & 0.08 & 8.59 & 0.15 & 2.02 & 2.59 \\
\hline
\end{tabular}

Lime Saturation Factor $(\mathrm{LSF})=\mathrm{CaO} /\left(2.8 \mathrm{SiO}_{2}+1.18 \mathrm{Al}_{2} \mathrm{O}_{3}+0.65 \mathrm{Fe}_{2} \mathrm{O}_{3}\right)$

Silicate Ratio $(\mathrm{SM})=\mathrm{SiO}_{2} /\left(\mathrm{Al}_{2} \mathrm{O}_{3}+\mathrm{Fe}_{2} \mathrm{O}_{3}\right)$.

Aluminum Ratio $(\mathrm{AM})=\left(\mathrm{Al}_{2} \mathrm{O}_{3} /\left(\mathrm{Fe}_{2} \mathrm{O}_{3}\right)\right.$ 
The silica ratio (also known as the Silica Modulus) is defined as: $\mathbf{S M}=\mathbf{S i O}_{2} /\left(\mathbf{A l}_{2} \mathbf{O}_{3}+\right.$ $\mathrm{Fe}_{2} \mathrm{O}_{3}$ ). A high silica ratio means that more calcium silicates are present in the clinker and less celite $\left(3 \mathrm{CaO} . \mathrm{Al}_{2} \mathrm{O}_{3}\right)$ and ferrite (3CaO. $\mathrm{Al}_{2} \mathrm{O}_{3} \cdot \mathrm{Fe}_{2} \mathrm{O}_{3}$ ). SM exits $\leq 3$ for most cement products and is typically between 2.0 and 2.8 .

The alumina ratio (also known as the Alumina Modulus) is defined as: $\mathbf{A M}=$ $\left(\mathbf{A l}_{2} \mathbf{O}_{3} /\left(\mathbf{F e}_{2} \mathbf{O}_{3}\right)\right.$. This determines the potential relative proportions of celite $\left(3 \mathrm{CaO} \cdot \mathrm{Al}_{2} \mathrm{O}_{3}\right)$ and ferrite $\left(3 \mathrm{CaO} \cdot \mathrm{Al}_{2} \mathrm{O}_{3} \cdot \mathrm{Fe}_{2} \mathrm{O}_{3}\right)$ phase in the clinker. It is $\leq 2$ and the preferable range should be between 1.6 and 1.8 because lower values leads to lower clinkering temperature. Moreover, an increase in clinker AM means there will be proportionally more celite $\left(3 \mathrm{CaO} \cdot \mathrm{Al}_{2} \mathrm{O}_{3}\right)$ and less ferrite in the clinker.

The other controlling factors are the level of the $\mathrm{MgO}, \mathrm{SO}_{3}$ and $\mathrm{Cl}$. If higher levels of $\mathrm{SO}_{3}$ and $\mathrm{Cl}$ exit in the raw materials, troubles in the kiln during burning are expected. The $\mathrm{MgO}$ content should not exceed 5\% in raw materials as it forms small crystals of periclase which are slowly hydrated in the cement or concrete expand and cause cracking. Clays usually contain limited amounts of $\mathrm{Na}_{2} \mathrm{O}, \mathrm{K}_{2} \mathrm{O}, \mathrm{SO}_{3}$ and $\mathrm{Cl}<0.3,2 \%$ and $0.015 \%$ respectively, it would be suitable for manufacturing cement, while silica must not exceed three times the percentages of $\mathrm{Al}_{2} \mathrm{O}_{3}+\mathrm{Fe}_{2} \mathrm{O}_{3}$ as previously mentioned by Youssef (1994).

Table 5: The average chemical analysis of limestone and clay samples in the studied area.

\begin{tabular}{|c|c|c|c|c|c|c|c|c|}
\hline & \multicolumn{4}{|c|}{$\begin{array}{c}\text { Limestone } \\
\text { (Gebel El Ramliya) }\end{array}$} & \multicolumn{4}{|c|}{$\begin{array}{c}\text { Kaolinitic Clay } \\
\text { (Abu Darag) }\end{array}$} \\
\hline & Min & Max & $\begin{array}{l}\text { Mean } \\
(n=55)\end{array}$ & Std & Min & Max & $\begin{array}{l}\text { Mean } \\
(n=26)\end{array}$ & Std \\
\hline $\mathrm{SiO}_{2}$ & 0.05 & 2.11 & 0.39 & 0.41 & 57.07 & 64.34 & 61.60 & 2.04 \\
\hline $\mathrm{Al}_{2} \mathrm{O}_{3}$ & 0.05 & 1.05 & 0.17 & 0.16 & 12.84 & 22.97 & 17.15 & 2.80 \\
\hline $\mathrm{Fe}_{2} \mathrm{O}_{3}$ & 0.02 & 0.59 & 0.11 & 0.11 & 5.02 & 9.25 & 7.07 & 0.96 \\
\hline $\mathrm{MgO}$ & 0.08 & 8.18 & 1.07 & 1.49 & 0.16 & 0.35 & 0.24 & 0.05 \\
\hline $\mathrm{CaO}$ & 43.04 & 56.00 & 52.92 & 2.66 & 0.30 & 0.32 & 0.31 & 0.00 \\
\hline $\mathrm{Na}_{2} \mathrm{O}$ & 0.03 & 0.24 & 0.08 & 0.05 & 0.10 & 0.23 & 0.15 & 0.04 \\
\hline $\mathrm{K}_{2} \mathrm{O}$ & 0.01 & 0.11 & 0.02 & 0.02 & 0.44 & 0.76 & 0.60 & 0.07 \\
\hline $\mathrm{SO}_{3}$ & 0.03 & 0.53 & 0.13 & 0.10 & 0.02 & 1.70 & 0.20 & 0.33 \\
\hline $\mathrm{Cl}$ & 0.00 & 0.12 & 0.03 & 0.03 & 0.05 & 0.24 & 0.12 & 0.05 \\
\hline LOI & 38.08 & 44.24 & 42.76 & 1.20 & 8.59 & 15.54 & 12.08 & 2.06 \\
\hline $\mathrm{CaCO}_{3}$ & 76.19 & 99.13 & 93.68 & 4.70 & & & & \\
\hline LSF & 631 & 21994 & 7344 & 5226 & 0.15 & 0.16 & 0.16 & --- \\
\hline SM & 0.42 & 2.58 & 1.37 & 0.48 & 1.77 & 3.43 & 2.60 & 0.42 \\
\hline AM & 0.37 & 3.00 & 1.77 & 0.57 & 1.82 & 3.05 & 2.44 & 0.36 \\
\hline
\end{tabular}

Lime Saturation Factor $(\mathrm{LSF})=\mathrm{CaO} /\left(2.8 \mathrm{SiO}_{2}+1.18 \mathrm{Al}_{2} \mathrm{O}_{3}+0.65 \mathrm{Fe}_{2} \mathrm{O}_{3}\right)$ 


\section{Quantitative Chemical Analysis ofthe Limestone and Clay in the Area}

\section{Quantitative chemical analysis of the limestone samples}

The main component of limestone is the calcium carbonates $\left(\mathrm{CaCO}_{3}\right)$, which on heating dissociates into $\mathrm{CaO}$ and $\mathrm{CO}_{2}$. According to the data of Table 5 the average range of silica falls between $0.05 \%$ and $2.11 \%$; these contents are relatively high in some outcrops. The average ranges of alumina, iron oxide, lime and magnesia contents fall between $0.05-1.05 \%, 0.02-0.59 \%$, $43.04-56.00 \%$, and $0.08-8.18 \%$, respectively. The high level of magnesia oxide $(\mathrm{MgO})$ is related to the dolomitic bands in the lower part of the Mokattam group which consisting of observatory formation shallow marine dense medium bedded limestone with local chert and numulites. Obviously, the $\mathrm{MgO}$ content in some samples exceeds $5 \%$ in raw materials as it forms small crystals of periclase which are slowly hydrated in the cement or concrete expand and cause cracking. The high $\mathrm{MgO}$ content also led to reduce viscosity of liquid phase, quicker formation of Tricalcium Silicate (Alite phase: $\mathrm{C}_{3} \mathrm{~S}$ ) then decomposition into Belite $\left(\mathrm{C}_{2} \mathrm{~S}\right)$. The average ranges of alkalis, chloride and sulphate contents are ranging between 0.04 and $0.34 \%, 0.03 \%$, and $0.13 \%$; respectively. The average range of lime saturation factor (LSF) is between $631 \%$ and $21994 \%$. While silica modulus $(\mathrm{SM})$ is ranging between $0.42 \%$ and $2.58 \%$ and Alumina modulus (AM) between $0.37 \%$ and $3.00 \%$. The low silicate is mainly owing to the low contents of silica and low contents of alumina and iron oxides. The average total carbonate is between $76.19 \%$ and $99.13 \%$, while the non-carbonate constituents are around $1-15 \%$. The loss on ignition falls between $38.08 \%$ and $44.24 \%$. Accordingly, based on the above-mentioned factors and their ranges, the limestone in Gabel El Ramliya area are suitable for the cement industry due to the presence of low sulphate and chloride.

\section{Quantitative chemical analysis of the Abu Darag kaolinitic clay samples}

According to the data shown in Tables 4 and 5, the average range for contents of silica falls at (57.07 and 64.34\%), alumina (12.84 and $22.97 \%$ ), iron oxides (5.02 and 9.25\%), lime $(\sim 0.30 \%)$, magnesia ( 0.16 and $0.35 \%)$, alkalis $(0.56$ and $0.97 \%)$, chlorides (0.05 and $0.24 \%$ ) and sulphates (0.02 and $1.70 \%)$. The calculated average range of lime saturation factor (LSF) is between $0.15 \%$ and $0.16 \%$, these values are very low due to increasing silica, alumina and iron oxides contents. The average range of Silica Modulus (SM) exists between $1.77 \%$ and $3.43 \%$ and Alumina Modulus (AM) ranges between $1.82 \%$ and $3.05 \%$ and the loss on ignition falls between $8.59 \%$ and $15.54 \%$. For clay, the proportion of silica to iron oxide and alumina oxide ranges around 1.77 to 343 (average 2.60; within the recommended range), which are adequate for cement industry.

\section{The chemistry of the Raw Mix inthe Study Area}

Cements are generally combinations of limestone, shells or chalk, and shale, clay, sand or iron ore, usually mined from a quarry close to the plant. The basic chemical components of Portland Cement (PC) are calcium (Ca), silicon ( $\mathrm{Si}$ ), aluminum (Al) and iron (Fe). The percent of limestone and clay forming raw material mix varies from plant to another according to their material available, plant specifications or KPI's. By the way, cement raw materials percent are trials that meet some standard. It is usual that the limestone component is around $75 \%$ of the mix and the clay forms $\sim 25 \%$. Assuming that, at a round of previous rates of limestone and the clay are to be used for raw materials, it is supposed to be the limits of the important and influential elements, depending on the average chemical analysis of clay and limestone on the area as follows: the average lime content should be range between $38 \%$ and $42 \%$, while magnesia content falls between $0.68 \%$ and $3.55 \%$. The $\mathrm{MgO}$ will cause some operational problems if higher $\mathrm{MgO}$ content of limestone is used as 
corrective materials. The average alkalis $\left(\mathrm{K}_{2} \mathrm{O}+\right.$ $\mathrm{Na}_{2} \mathrm{O}$ ) content ranges between $0.42 \%$ and $1.13 \%$. The average chloride content lies between $0.15 \%$ and $0.71 \%$. The average sulphate content ranges between $0.12 \%$ and $0.52 \%$. The average lime saturation factor ranges between $81 \%$ and $110 \%$. The average silica modulus is ranging between $1.78 \%$ and $2.84 \%$; low silica modulus can be increased using corrective sands. The average alumina modulus falls between $1.22 \%$ and $1.94 \%$. Homogeneity of limestone is preferred to overcome the higher content of silica and magnesia.

\section{Conclusion}

The study area is located in the northern portion of the Eastern Desert of Egypt in the El Sukhna-Zaafrana old road on the Red Sea.

The foot slope of Gebel El Ramliya limestone. At Gebel El Ramliya, Middle Eocene Mokattam group consisting of Sannor (Observatory) Formation shallow marine dense medium bedded limestone with local chert and numulites. The average thickness of the limestone can be quarried ranging between $45 \mathrm{~m}$ and $70 \mathrm{~m}$ (Sannor Formation). In the area east of El Sukhna, large extension of limestone forming the cap rock of the plateau exists on both sides of the Cairo-Suez Road. The plateau extends from east of $\mathrm{El}$ Qattamiya to Red Sea, to around $100 \mathrm{~km}$ east west and almost $200 \mathrm{~km}$ north east. The limestone plateau in the area is dissected by number of wadis. The limestone plateau is made of massive carbonates with high content of chert bands. These wadis are W. Gharaba, and W. Hagul. The existence of these wadis makes it possible for limestone quarrying and sampling due to the height of plateau and its steep slope.

The limestone plateaus unconformably lies over the Permo-Triassic of Qiseib Formation that are made mainly of clays and kaolinitic clays. The sequence penetrated is an alternation of kaolintic clay beds and sandy clay beds with frequent sandstone intercalations. The koalintic clay is massive, indurated, dense, and light to greyishbrown in colour. The clay of this quality is semiconsolidated, kaolinitic, detrital, fine grained and dry. The sand is poor to well sorted, ranging in size from very fine to coarse sand, and even fine pebble size. The colors of the clay and sand varieties vary depending on the iron oxide contents.
The chemical analyses of 55 samples of limestone and 22 samples of clays that is made to evaluate their suitability for cement industries indicate that:

- Abu Darag red kaolinitic clay (RKC) in the area is suitable for cement industry. Where about 41 Million tonne of proven $\mathrm{Abu}$ Darag red kaolinitic clay (RKC) can be quarried for 30 years

- Field observation and measurements indicate that the average thickness of the limestone can be quarried ranging between $45 \mathrm{~m}$ and $70 \mathrm{~m}$ (Sannor Formation). About 200 Million tonne of proven limestones can be quarried for 50 years.

- The limestone of Sannor (Observatory) Formation is not suitable for cement industries with regard to the high content of chert bands and silica concretions.

- The limestone of Drunka Formation especially the upper most parts $(50-70 \mathrm{~m})$ satisfies all the requirements for the manufacturing of the Portland cement clinker.

\section{References}

Cement Sustainability Production Report (2011). Cement Production, http://www. wbcsdcement.org/index.php/aboutcement/cement-production

Hewlett, P.C. and Peter, C. (1997). Chemistry of Cement and Concert, Hewlett, P.C. (Ed.): Butterworth - Heinemann, 1053 pages.

Kosmatka, S., Kerhof, F.B. and Panarese W.O. (2002). Design and Control of Concrete Mixture, $14^{\text {th }}$ ed., Portland Cement Association, Skoki II.

Taylor, H.F.W. (1964). Cement Chemistry, Academic Press, London and New York, Vol. 1, 460 pages, Vol. 2, 442 pages.

Taylor, H.F.W. (1997). Cement Chemistry, $2^{\text {nd }}$ ed., Thomas Limited Pub., Telford, 459 pages.

Youssef, M. (1994). A Giant Consumer of Sedimentary Rocks: The Cement Industry, Sedimentlogical Lecture Season '5', Geol. Dept., Faculty Science, Ain Shams University, Cairo, Egypt. 
تقييم المواد الخام لتصنيع الأسمنت بمنطقة السخنه ــ الزعفرانه، الصحراء الثرقية ـ مصر

حسن زكريا حراز، محمد محمود حمدى، عبد السلام محمد ابوالعلا، إسلام محمد الحسيني

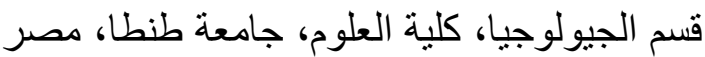

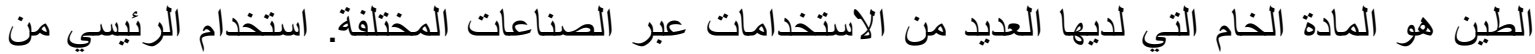

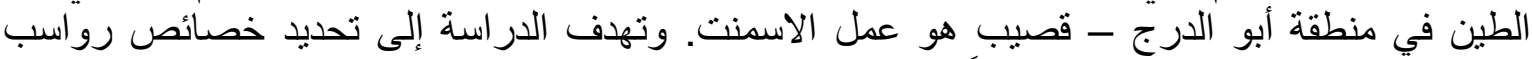

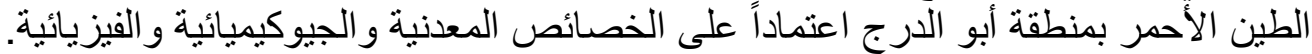

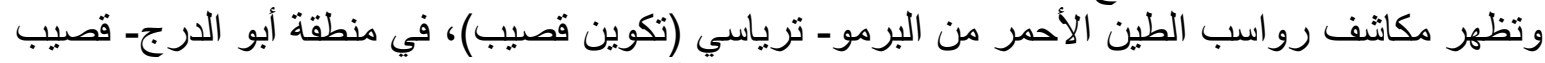

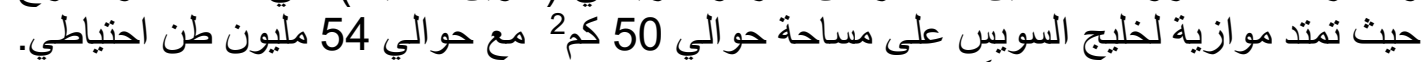

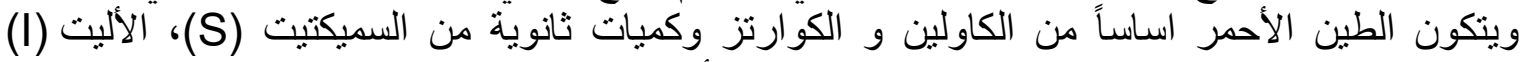

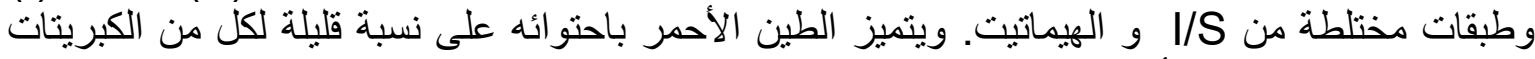

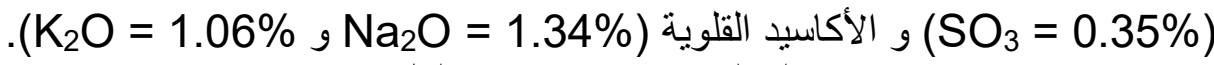

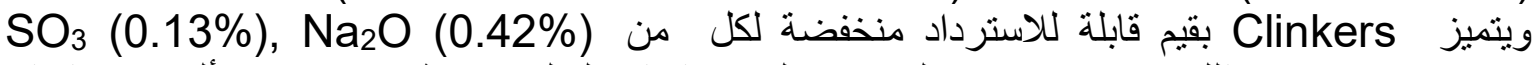

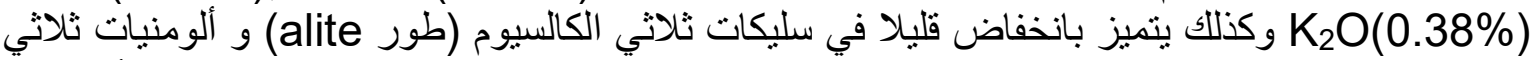

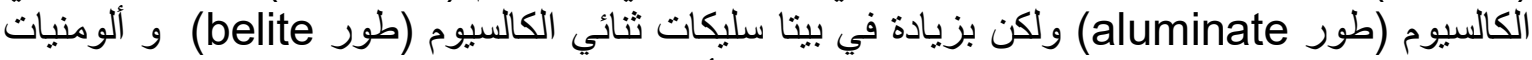

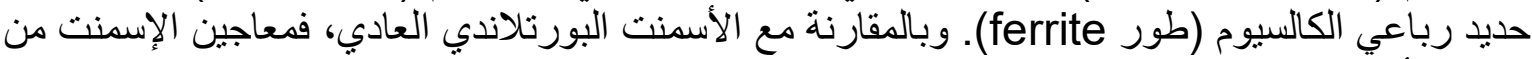

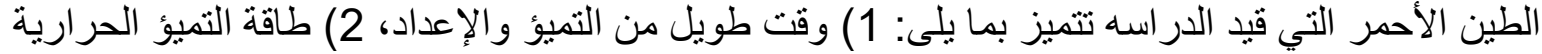

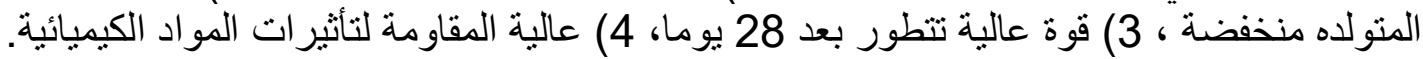

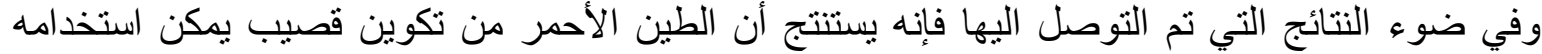
لإنتاج أنو اع خاصة من الأسمنت، مثل الاسمنت المقاوم للمو اد الكيميائية والأسمنت الكنخفض الكن الحرارة. 